\title{
Optimal investment policy for a company under inflation risk
}

\author{
Zhonghua Zhu \\ School of Management Science and Engineering, \\ Central University of Finance and Economics, Beijing, China
}

\begin{abstract}
In this paper, we consider the optimal investment control problem for a company who worries about inflation risk. We assume that the company is self-financing. The decision maker of the company can invest in a financial market consisting of two assets: one risk-free asset, one risky asset. Our purpose is to find the impacts of inflation on optimal investment policy. With the objective of maximizing the CRRA utility of terminal wealth, the closed-form solutions of the optimal investment policy are obtained by solving HJB equations. We find that the optimal investment policy is affected by the correlation coefficient between the price of risky asset and price index.
\end{abstract}

Keywords: Optimal investment policy, Inflation risk, HJB equations, CRRA utility, risky asset

\section{INTRODUCTION}

The optimal investment problem has been studied for decades. For example, Markowitz (1952) considered the portfolio selection problem with the relevant beliefs about future performances and ends with the choice of portfolio. Merton $(1969,1971)$ investigated the optimal investment policies in a well-known continuous-time model. Many researchers studied the investment strategies with maximizing utility of terminal wealth for a variety of utility functions see Karatzas (1989) and many others. Browne (1995) analyzed the optimal investment policies with maximizing exponential utility of terminal wealth and minimizing the probability of ruin. Milevsky and Young (2007) studied the optimal investment policy for a retiree with stochastic time of death. It is obvious that the above researches do not consider the inflation risk. Other studies on optimal investment problem see Browne (1999,2000a,2000b), Maenhout (2004) , Pliska and Ye (2007), Young and Zhang (2016), Bayraktar and Zhang (2015), Bayraktar and Young (2007).

In our work, we focus on the optimal investment policy for a self-financing company with consideration of inflation risk. Without loss of generality, we assume that there are only one risk-free asset and one risky asset in the financial market. And the decision maker of the company can choose to invest one of them. The inflation risk is modeled as a price index.

The purpose of the company is to achieve a maximizing utility of a given time (terminal time). By the optimal principle of dynamic programming, the closed-form solutions of the optimal investment and value function are obtained. Furthermore, we find that the optimal investment policy is mainly affected by the correlation coefficient between the price of risky asset and price index.

The remainder of this paper is organized as follows. Section 2 describes the surplus process of the company, then gives the value function. In Section 3, by employing the dynamic programing principle, we derive the HJB equation which satisfied by the value function. And the closedform solutions of the optimal policy and the value function are obtained. In Section 4, we give some numerical examples to illustrate the impacts of the inflation risk. 


\section{THE MODEL}

In this section, we describe the surplus process of the company in which the decision maker of the company can invest into the financial market. The inflation risk is described by a price index model.

The price process $P_{0}(t)$ of the risk-free asset is

$$
\frac{\mathrm{d} P_{0}(t)}{P_{0}(t)}=r \mathrm{~d} t
$$

where $r>0$ is a risk-free interest rate. And the price process $P_{1}(t)$ of the risky asset is described by a geometric Brownian motion

$$
\frac{\mathrm{d} P_{1}(t)}{P_{1}(t)}=\mu \mathrm{d} t+\sigma \mathrm{d} B(t)
$$

where $\mu>0$ and $\sigma>0$ are constants, $B(t)$ is a $\mathrm{d} I_{t}$ standard Brownian motion.

Denote the proportion of the wealth invested in risky asset at time $t$ by $\pi_{t}$, and $1-\pi_{t}$

is the proportion of the wealth to invest in risk-free asset, then the nominal surplus process $X_{t}$ of the self-financing company is

$$
\mathrm{d} X_{t}=X_{t} \pi_{t}(\mu \mathrm{d} t+\sigma \mathrm{d} B(t))+X_{t}\left(1-\pi_{t}\right) r \mathrm{~d} t, X_{0}=X,
$$

where $x$ is the initial wealth of the company.

Assume that the inflation risk is controlled by a price index. And the price index $I_{t}$ is describe by

$$
\frac{\mathrm{d} I_{t}}{I_{t}}=\mu_{I} \mathrm{~d} t+\sigma_{I} \mathrm{~d} B_{I}(t)
$$

where $\mu_{I}>0$ and $\sigma_{I}>0$ are constants, $B_{I}(t)$ is a standard Brownian motion. The correlation relationship of the financial market and the price index is described by $\operatorname{Cov}\left(B(t), B_{I}(t)\right)=\rho t$. Obviously, the inflation-adjusted surplus process is $\bar{X}_{t}=\frac{X_{t}}{I_{t}}$. By

Itô lemma, we have:

$$
\begin{aligned}
& \mathrm{d} \bar{X}_{t}=\frac{1}{I_{t}} \mathrm{~d} X_{t}+\frac{-X_{t}}{\left[I_{t}\right]^{2}} \mathrm{~d} I_{t}-\frac{1}{\left[I_{t}\right]^{2}} \mathrm{~d} I_{t} \mathrm{~d} X_{t}+\frac{-X_{t}}{\left[I_{t}\right]^{3}} \mathrm{~d} I_{t} \mathrm{~d} I_{t} \\
& =\bar{X}_{t}\left[\left(\mu \pi_{t}+\left(1-\pi_{t}\right) r-\mu_{I}-\sigma \sigma_{I} \rho+\sigma_{I}{ }^{2}\right) \mathrm{d} t+\pi_{t} \sigma \mathrm{d} B(t)-\sigma_{I} \mathrm{~d} B_{I}(t)\right] .
\end{aligned}
$$

Let $T$ denote the terminal time. The decision maker of the company wants to find an optimal investment in order to maximize the expected utility of the terminal wealth. Thus, the value function in this paper can be represented as 


$$
V(t, x)=\sup _{\pi_{t}} E_{t, x}\left[U\left(X_{T}\right)\right] \text {, }
$$

where $E_{t, x}[\cdot]=E\left[\cdot \mid X_{t}=x\right]$ is a conditional expectation, $U(\cdot)$ is a utility function, and the boundary condition can be obtained by $V(T, x)=U(x)$.

\section{OPTIMAL INVESTMENT POLICY}

In this section, we use the dynamic programming principle to obtain the optimal investment policy. We firstly give a definition of the set that contains all admissible policies.

Definition 3.1. We say that $\Pi=\left\{\pi_{t}, 0 \leq t \leq T\right\}$ is the set of admissible policies, if the process $-\infty<\pi_{t}<\infty, T<\infty$ (i.e., both short-selling and borrowing at risk-free rate are allowed) and

$$
E \int_{0}^{T} \pi_{s}^{2} \mathrm{~d} s<\infty
$$

In this paper, the utility function is specified by the CRRA utility function

$$
U(x)=\frac{x^{1-\gamma}}{1-\gamma}, x>0, \gamma>1 \text {, }
$$

where $\gamma$ is the relative risk aversion.

According to the technique of dynamic programing principle, if $V(t, x) \in \mathbb{C}^{1,2}$, then the value function satisfies the Hamilton-Jacobi-Bellman (HJB) equation (see Fleming and Soner (2006))

$$
\begin{aligned}
& \sup _{\pi_{t} \in \Pi}\left\{V_{t}+\left[\mu \pi_{t}+\left(1-\pi_{t}\right) r-\mu_{I}-\sigma \sigma_{I} \rho+\sigma_{I}^{2}\right] X V_{x}\right. \\
& \left.+\frac{1}{2}\left[\pi_{t} \sigma^{2}+\sigma_{I}^{2}-2 \pi_{t} \sigma \sigma_{I} \rho\right] x^{2} V_{x X}\right\}=0
\end{aligned}
$$

and the boundary condition is $V(T, x)=U(x)$.

Theorem 3.1. (Verification Theorem) If $v(t, x) \in \mathbb{C}^{1,2}$ is the solution to HJB equation (8) with the boundary condition $v(T, x)=U(x)$, then $v(t, x)=V(t, x)$.

\section{Proof}

The proof of the verification theorem can be given by the usual technique. And it is similar to the result of the Browne (1995). So we omit it here.

With the form of the boundary condition, we can conjecture the value function satisfies the following form

$$
V(t, x)=H(t) \frac{x^{1-\gamma}}{1-\gamma},
$$


where $H(t)$ is a function of $t$, and $H(T)=1$. Thus, we can obtain that

$$
\begin{aligned}
& V_{t}(t, x)=H^{\prime}(t) \frac{x^{1-\gamma}}{1-\gamma} \\
& V_{x}(t, x)=H(t)_{X^{-\gamma}} . \\
& V_{X X}(t, x)=-\gamma H(t)_{X^{-\gamma-1}}
\end{aligned}
$$

Substituting (10) into (8), we have

$$
\begin{aligned}
& \sup _{\pi_{t} \in \Pi}\left\{H^{\prime}(t) \frac{1}{1-\gamma}+\left[\mu \pi_{t}+\left(1-\pi_{t}\right) r-\mu_{I}-\sigma \sigma_{I} \rho+\sigma_{I}^{2}\right] H(t)\right. \\
& \left.-\frac{1}{2}\left[\pi_{t}^{2} \sigma^{2}+\sigma_{I}{ }^{2}-2 \pi_{t} \sigma \sigma_{I} \rho\right] \gamma H(t)\right\}=0 .
\end{aligned}
$$

By the first condition, we obtain the optimal investment policy can be written as

$$
\pi_{t}^{*}=\frac{\mu-r+\sigma \sigma_{I} \rho \gamma}{\sigma^{2} \gamma} .
$$

Putting (12) into (11), we have

$$
\begin{aligned}
& H^{\prime}(t) \frac{1}{1-\gamma}+\frac{\left[\mu-r+\sigma \sigma_{I} \rho \gamma\right]^{2}}{2 \sigma^{2} \gamma} H(t) \\
& +\left[r-\mu_{I}-\sigma \sigma_{I} \rho+\sigma_{I}^{2}-\frac{\sigma_{I}^{2} \gamma}{2}\right] H(t)=0
\end{aligned}
$$

Simplifying (13), we have

$$
\frac{H^{\prime}(t)}{H(t)}=(1-\gamma)\left[-\frac{\left(\mu-r+\sigma \sigma_{I} \rho\right)^{2}}{2 \sigma^{2} \gamma}-\left(r-\mu_{I}-\sigma \sigma_{I} \rho+\sigma_{I}^{2}-\frac{\sigma_{I}^{2} \gamma}{2}\right)\right],
$$

By the boundary condition $H(T)=0$, we obtain that

$$
H(t)=\exp \left\{(1-\gamma)\left[-\frac{\left(\mu-r+\sigma \sigma_{I} \rho\right)^{2}}{2 \sigma^{2} \gamma}-\left(r-\mu_{I}-\sigma \sigma_{I} \rho+\sigma_{I}^{2}-\frac{\sigma_{I}^{2} \gamma}{2}\right)\right](t-T)\right\} .
$$

With the above process, we have obtained the solutions to the optimal investment policy and the value function indeed, which can be concluded as the following theorem.

Theorem 3.2. For the problem (6) with utility function (7), we can obtain the closed-form solutions for optimal investment policy and value function:

The optimal investment policy is

$$
\pi_{t}^{*}=\frac{\mu-r+\sigma \sigma_{I} \rho \gamma}{\sigma^{2} \gamma}
$$


The value function is

$$
V(t, x)=H(t) \frac{x^{1-\gamma}}{1-\gamma}
$$

$$
\text { where } H(t)=\exp \left\{(1-\gamma)\left[-\frac{\left(\mu-r+\sigma \sigma_{I} \rho\right)^{2}}{2 \sigma^{2} \gamma}-\left(r-\mu_{I}-\sigma \sigma_{I} \rho+\sigma_{I}^{2}-\frac{\sigma_{I}^{2} \gamma}{2}\right)\right](t-T)\right\} \text {. }
$$

Remark 1. From Theorem 3.2, we can see that the impact of the inflation risk (price index) on optimal investment policy mainly derived by the correlation relationship of the financial market and the price index.

\section{NUMERICAL EXAMPLES}

In order to illustrate the impacts of inflation risk on the optimal investment policy, we would like to give some numerical examples. Unless otherwise stated, we take the following values for the parameters:

- For the financial market: $r=0.04, \mu=0.08, \sigma=0.2$.

- For the inflation risk: $\sigma_{I}=0.1$.

- For others: $\gamma=2, \rho=0.3$.

We present the impact of the $\rho, \gamma, \sigma_{I}$ on the optimal investment policy in Figure 1,2, 3 , respectively.

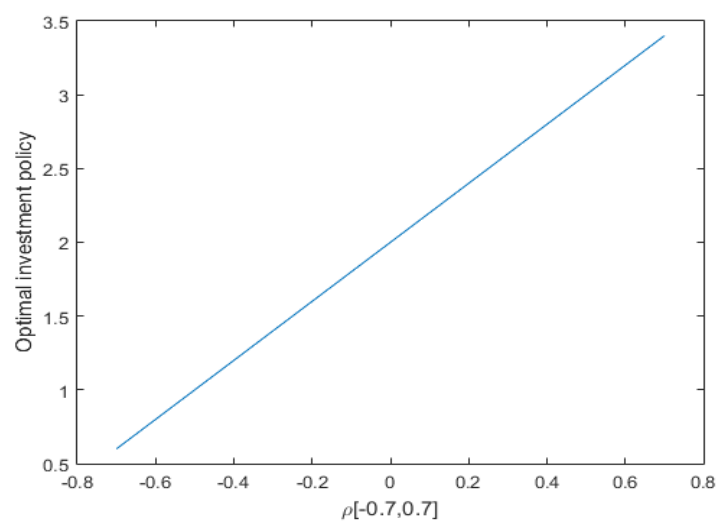

Figure 1: The impact of $\rho$ on optimal investment policy

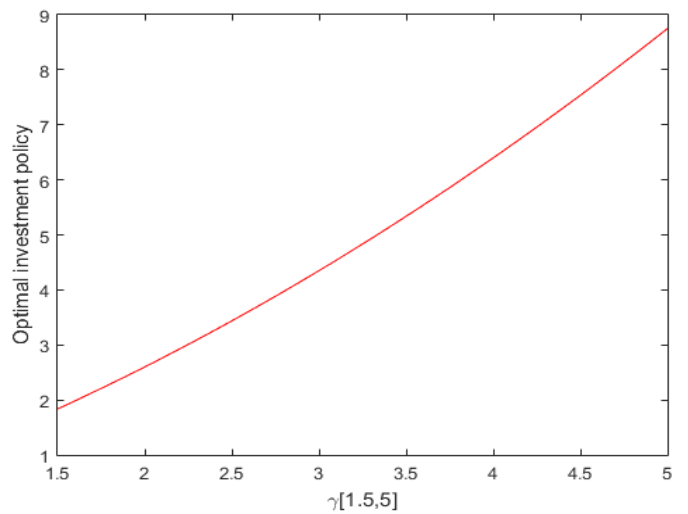

Figure 2: The impact of $\gamma$ on optimal investment policy 


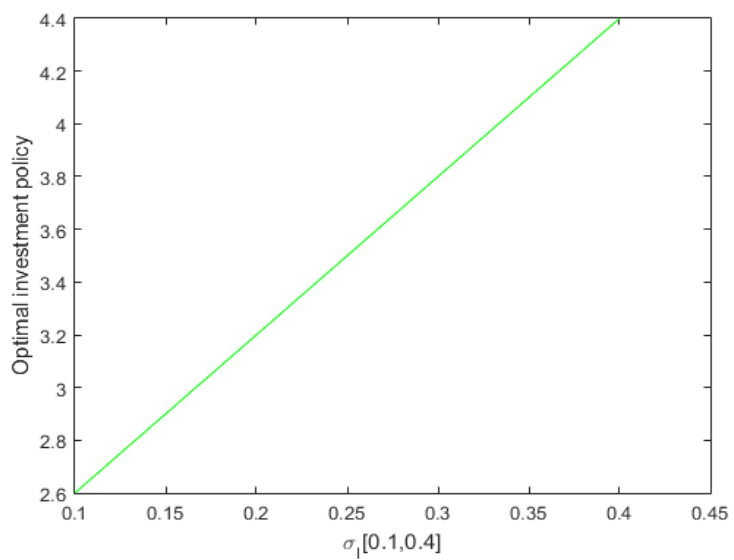

Figure 3: The impact of $\sigma_{I}$ on optimal investment policy

\section{References}

Bayraktar, E., Young, V.R., Minimizing the probability of lifetime ruin under borrowing constraints. Insurance Mathematics and Economics, 2007, 41(1):196-221.

Bayraktar, E., Zhang, Y., Minimizing the probability of lifetime ruin under ambiguity aversion. SIAM J. Control Optim., 2015, 53(1):58-90.

Browne, S., Optimal investment policies for a firm with a random risk process: exponential utility and minimizing the probability of ruin. Mathematics of Operations Research, 1995, 20:937--958.

Browne, S., Beating a moving target: Optimal portfolio strategies for outperforming a stochastic benchmark. Finance and Stochastics, 1999, 3(3):275-294.

Browne, S., Risk-constrained dynamic active portfolio management. Management Science, 2000a, 46(9):11881199.

Browne, S., Stochastic differential portfolio games. Journal of Applied Probability, 2000b, 37(1):126-147.

Fleming, W.H., Soner, M.. Controlled Markov Processes and Viscosity Solutions, 2nd edition. Springer, 2006, New York.

Karatzas, I., Optimization problems in the theory of continuous trading. SIAM J. Control. And Optim. 1989, 7:12211259.

Maenhout, P.J., Robust portfolio rules and asset pricing. Review of Financial Studies, 2004, 17(4):951-983.

Markowitz, H.M., Portfolio Selection. Journal of Finance, 1952, 7:77-91.

Merton, R.C., Lifetime portfolio selection under uncertainty: The continuous-time case. Review of Economics and Statistics, 1969, 51(3):247-257.

Merton, R.C., Optimum consumption and portfolio rules in a continuous-time model. Journal of Economic Theory, 1971, 3(4):373-413.

Pliska, S.R., Ye, J., Optimal life insurance purchase and consumption/investment under uncertain lifetime. Journal of Banking and Finance, 2007, 31(5):1307-1319.

Young, V. R., Zhang, Y., Lifetime ruin under ambiguous hazard rate. Insurance Mathematics and Economics, 2016, 70:125-134. 